\title{
Probability based Extended Direct Attribute Prediction
}

\author{
Manju \\ Research Scholar, \\ Department of computer science, \\ Baba Mastnath University, Rohtak
}

\author{
Ankit Kumar, PhD \\ Assistant professor, \\ Department of computer science, \\ Baba Mastnath University, Rohtak
}

\begin{abstract}
This paper studies the object recognition along with the direct and indirect attribute prediction. The direct attribute prediction technique has been extended by using the probability based formulae. Moreover, information gain is also used to classify the object into different categories. The information gain is determined by using the entropy. The implementation of the work and comparison with existing DAP technique over YAHOO and pascal dataset signifies the effectiveness of the work.
\end{abstract}

\section{Keywords}

Dap, Iap, Probability, Sensitivity, Specificity.

\section{INTRODUCTION}

Object acknowledgment is performed by coordinating the key purposes of the specimen with the key focuses put away in database of preparing tests. The uncertain elements can results wrong matches. Learning-based techniques for perceiving objects [2] in regular pictures have gained vast ground throughout the most recent years.

Applications for recognition systems are manifold and their number is steadily growing with the rapid improvements in signal acquisition and storage capabilities. Different types of sensors are almost omnipresent and provide an abundance of data that needs to be processed. A recent space shuttle mission to map the surface of the earth produced more than 12 Terabytes of data, corresponding to over 20,000 CDs or approximately the content of the Library of Congress. More and more large databases of texts and images are becoming available publically on the internet [3-5]. CNN plans to make its entire archive searchable over the internet, while the New York Times has already done so. There is no possibility of processing such large amounts of data by hand. Therefore, automatic tools that understand or at least extract useful information from images are needed. Object recognition would be key. Interaction with machines is often difficult and cumbersome, if not impossible, unless these machines understand something about their environment, in particular the objects and creatures they interact with. In order to navigate in any environment, vision is the most important sensory modality. In interactions between humans, visual input is almost as important as auditory information. This suggests that vision should play a crucial role in humanmachine interactions. Examples include computer interfaces in general, computer games, interactions with appliances or automobiles, as well as the problem of surveillance. Automobiles now have vision-based systems that drive autonomously or at least assist the driver in keeping up with the changes in the outside environment. It is of great importance that these systems be able to detect objects such as roads, pedestrians, traffic signs, and other vehicles [6]. Another rapidly expanding area in computer vision is medical image processing. Here, machines that can accurately detect objects such as bones, organs, boundaries between different types of tissues, or tumors would be of great practical usefulness. Such biological objects lend Vital to comprehension pictures is the issue of perceiving articles in pictures. People can perceive questions easily and are seldom even mindful of the adjustments in an item's appearance that happen, for instance, because of alters in review course or a shadow being thrown over the article [7]. We likewise promptly gather occasions of items, for example, autos, confronts, shoes, or houses into a solitary article class and disregard the contrasts between the individual individuals. In the meantime, we can in any case separate on a subunmitigated level, expressing, for instance, "Here comes Peter, strolling my dog!". On the other hand, everybody who has ever managed a PC has unavoidably encountered that even the littlest change in the data gave to a PC can, and frequently makes, all the distinction on the planet. Instructing a machine to perceive items is about showing it which contrasts in the crude picture data matter and which don't. The center of this theory is on the issue of learning principled representations of articles naturally from tactile signs, and on how such representations can be utilized to identify objects. What precisely do we mean by "articles" and "protest classes or classifications?" It is difficult to give a formal definition, since the term is utilized as a part of an exceptionally expansive way. We consider an item to be a part of, or token in, a tactile sign. The exact representation of the item inside the sign can experience changes, for example, scaling, interpretation, or different miss-happenings, or it can be defiled by commotion or be somewhat blocked. These progressions offer ascent to a whole accumulation or class of signs which would all be able to even now be connected with the first protest. Objects in signs regularly compare to physical items in this present reality environment from which the signs have been recorded. This is the situation for articles in pictures of normal scenes. In any case, articles can likewise be characterized exclusively in the universe of signs [8]. For instance, an example in a recording of a birdsong or an in recording of neural movement may be characterized as an item. Classes of items are accumulations of articles that are comparative. The likeness may be founded on abnormal state subjective standards, e.g., on account of the class of seats. For this situation an acknowledgment framework may need to epitomize or build up some comprehension of these ideas so as to recognize objects of the class. In a more tractable situation, the similitude to a great extent shows itself at the level of the sign representation. For instance, bits of the signs of two distinct articles from the same class could be indistinguishable. We utilized the expression "tactile sign" above, since we trust that our techniques are not confined to picture information. They have been effectively connected by Burl et al. to the issue of acknowledgment of transcribed characters and words, in view of a representation of the ink follow as far as point directions after some time. We have additionally had starting accomplishment in characterizing neural spike trains speaking to data about scents in the olfactory knob of the grasshopper[9-10]. 


\section{ATTRIBUTE CLASSIFIER}

In making a classifier for a specific quality, we could essentially extricate a wide range of low-level components from the entire protest, and let a classifier make sense of which are vital for the errand and which are definitely not. Quality classifiers $C_{-} i$ are fabricated utilizing an administered learning approach. Preparing requires an arrangement of marked positive and negative pictures for every property. The objective is to manufacture a classifier that best arranges this preparation information by picking a suitable subset of the list of capabilities F (I). Every classifier's execution is assessed utilizing cross approval. The components utilized as a part of the classifier with the most elevated cross-approval exactness are added to the yield set. We keep including highlights until the precision quits moving forward. At the point when no preparation tests are accessible the accompanying procedure can be utilized.

\subsection{Attribute-Based Classification}

The classification of any object based on its feature is the only solution to the distinct features problem while testing and training. Two techniques exist in literature for the multi class classification based on features are DAP (Direct Attribute Prediction) and IAP ( Indirect attribute prediction).

\subsubsection{DAP (Direct Attribute Prediction)}

It utilizes a layer to differentiate between the objects and the label of objects. The layer in-between two layers is used in the training process to produce deterministic label of the objects. These deterministic labels are used in the testing phase to determine the features of the sample object as shown in fig 1 .

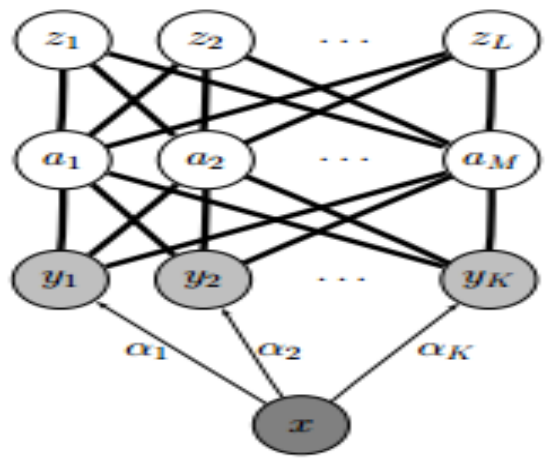

Figure 1: DAP (Direct Attribute Prediction) [2]

\subsubsection{IAP (Indirect attribute prediction)}

In this method, attributes are used for the information travel as in the Dap but the in-between layer among two label layers, one layer is the labels which are given in the training time while other is layer of unknown label. This technique is similar to the simple training process during training while the testing uses a feature layer to produce the label.

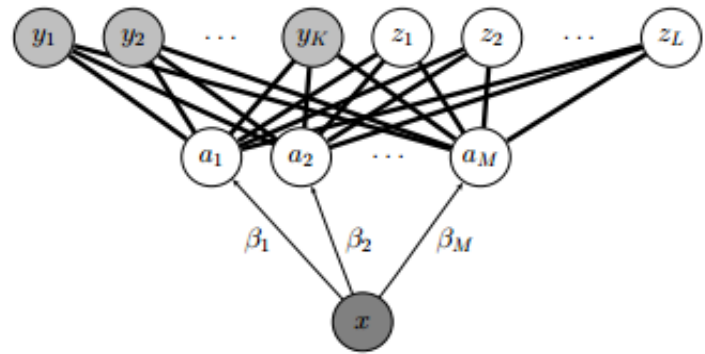

Figure 2 : Indirect Attribute Prediction (IAP) [2]
The figure 2 shows the IAP i.e. indirect attribute prediction.

\section{PROBABILITY BASED FORMULA TO EXTEND THE DAP}

The attributes in class can be either active or inactive. Suppose $\mathrm{F}_{\mathrm{q}}=\left(\mathrm{f}_{1}^{\mathrm{q}}, \ldots \ldots . \mathrm{f}_{\mathrm{m}}^{\mathrm{q}}\right)$ be a vector of binary associations $\mathrm{f}_{\mathrm{m}}^{\mathrm{q}} \in\{0,1\}$ between attributes $\mathrm{f}_{\mathrm{m}}$ and training object classes q.

A classifier for feature (attribute) fm, trained by classification all objects having different classes for which $\mathrm{f}_{\mathrm{m}}^{\mathrm{q}}=1$ as positive and the rest as negative training examples, can provide an estimate of the posterior $\mathrm{P}\left(\mathrm{f}_{\mathrm{m}} \mid \mathrm{p}\right)$ of that attribute being present in object $\mathrm{p}$.

Mutual independence acquiesces $P(f \mid p)=\prod_{m=1}^{M} P\left(f_{m} \mid p\right)$ for different attributes.

To regulate and convey features information to an unidentified category $\mathrm{r}$, we take a binary vector fr for which $p(f \mid r)=1$ for $f=f r$ and 0 otherwise.

The posterior probability of class $r$ being present in object $p$ is then obtained by marginalizing over all possible attribute associations a, using Bayes' rule

$$
\begin{gathered}
p\left(r \mid f^{r}\right)=\frac{P\left(f^{r} \mid r\right) P(r)}{P\left(f^{r}\right)}=\frac{P(r)}{P\left(f^{r}\right)} \\
P(r \mid p)=\sum_{f \in\{0,1\}^{M}} P(r \mid f) P(f \mid p)=\frac{P(r)}{P\left(f^{r}\right)} \prod_{m=1}^{M} P\left(f_{m} \mid p\right) f_{m}^{r}(1)
\end{gathered}
$$

Assuming identical class priors $\mathrm{P}(\mathrm{r})$ and a factorial distribution for $\left.P(f)=\prod_{m=1}^{M} P f_{m}\right)$, we obtain

$$
\mathrm{P}(\mathrm{r} \mid \mathrm{p}) \propto \prod_{\mathrm{m}=1}^{\mathrm{M}}\left(\frac{\mathrm{P}\left(\mathrm{f}_{\mathrm{m}} \mid \mathrm{p}\right)}{\mathrm{P}\left(\mathrm{f}_{\mathrm{m}}\right)}\right) \mathrm{f}_{\mathrm{m}}^{\mathrm{r}}
$$

Trait priors can be approximated by experimental means over the training classes (instructional courses) $\mathrm{P}\left(\mathrm{f}_{\mathrm{m}}\right)=$ $\frac{1}{\mathrm{~T}} \sum_{\mathrm{t}=1}^{\mathrm{T}} \mathrm{f}_{\mathrm{m}}^{\mathrm{qt}}$.

Classifying an object $\mathrm{p}$ according to test classes $\mathrm{r}_{\mathrm{L}}$ uses MAP prediction:

$f(x)=\max _{b=1, \ldots, L} \prod_{m=1}^{M}\left(\frac{P\left(f_{m} \mid p\right)}{p\left(f_{m}\right)}\right) f_{m}^{r b}$

The formulae can be extended in terms of the attributes. The attributes can have the probability between 0 and 1 . Suppose an object $p$ has $n$ attributes say $f 1, f 2, \ldots .$. fn. Then each attribute will have contribution to categorize the object the probability 0 means the attribute doesn't contribute towards its classification while the probability 1 means the attribute contributes completely.

The Bayes' theorem can also be applied on the attributes of the object as follow:

$$
P\left(r \mid f^{r}\right)=\frac{P\left(f^{r} \mid z\right) P(r)}{P\left(f^{r}\right)}=\frac{P(r)}{P\left(f^{r}\right)}
$$

While

$$
P(r \mid p)=\sum_{f \in\{0,1\}^{M}} P(r \mid f) P(f \mid p)=\frac{P(r)}{P\left(f^{r}\right)} \prod_{m=1}^{M} P\left(f_{m} \mid p\right) f_{m}^{r}(1)
$$

Two attributes may contribute similar then

$$
P(r \mid p) \propto \prod_{m=1}^{M}\left(\frac{P\left(f_{m} \mid p\right)}{P\left(f_{m}\right)}\right) \mathrm{f}_{m}^{r}
$$


These formulae can be applied to DAP as well as on IAP to extend the classification.

This probability base contribution will help to select the attributes from a cluster of attributes. Initially a group of attributes are available for the classification purpose. The group $\mathrm{f}$ attributes can be ivied in to cluster of attributes on the basis of their contribution in the classification. These attributes then arranged in a decreasing order. This order classify the objects by using the decision tree phenomena. The decision tree phenomena is as follow:

Compute Class frequency by using the probability formula.

Then if cli=1then create tree with one node

Otherwise, for each attribute in attribute list calculate gain ratio by using

$$
\text { gain }=\operatorname{info}(\mathrm{T})-\sum_{\mathrm{i}-1}^{\delta} \frac{\left|\mathrm{T}_{\mathrm{i}}\right|}{|\mathrm{T}|} \times \operatorname{info}\left(\mathrm{T}_{\mathrm{i}}\right)
$$

here the info is the ratio of probability, Where $\mathrm{T}$ is the total set of cases and c denotes classes.

The feature having most extreme pick up say $\mathrm{N}$ If $\mathrm{N}$ is persistent then discover edge that signifies the best estimation of the entire preparing set.

Make a Node and characterize the information on premise of quality $\mathrm{N}$ and expel the trait from ascribe list and go to beginning. This procedure arranges the picture on the premise of the chose properties.

\section{RESULTS AND DISCUSSION}

\subsection{Dataset Used}

This work is analyzed on two datasets one is the animal with attribute (dataset 1) and the other is Pascal-a-yahoo dataset( dataset 2). The details of these dataset are given in [2]. The implementation is done using the MATLAB and corresponding results on these datasets are discussed in further subsections.

\subsection{Performance Evaluation Parameters}

The performance of the algorithm is analyzed by using various parameters discussed below with their respective values are Accuracy, specificity and sensitivity.

\subsection{Result Analysis}

The analysis of the parameter discussed in previous section for the described two datasets has been given in the table 1 and table 2. The table 1 shows the comparison of the accuracy for the datasets. The comparison of the specificity and sensitivity is shown graphically using the figure 4 and 5 . The improvement in the parameter can be easily observed.

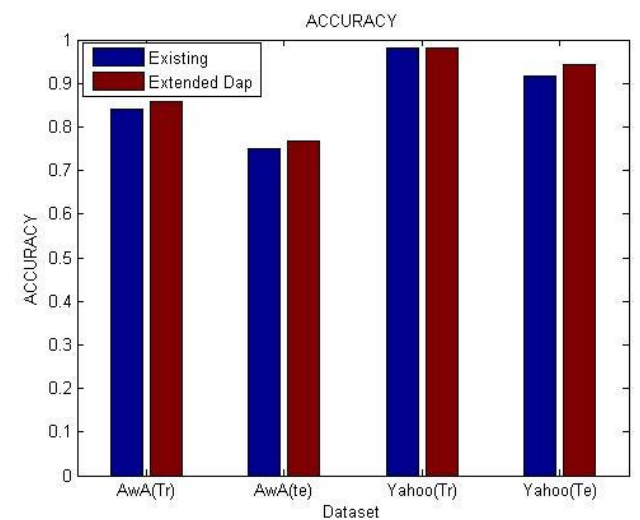

Fig 3: Comparison of Accuracy

The figure 3 shows the comparison of the accuracy values for the existing, extended Dap and the proposed algorithm over described datasets. The improvement in the accuracy can be analyzed by the graph.

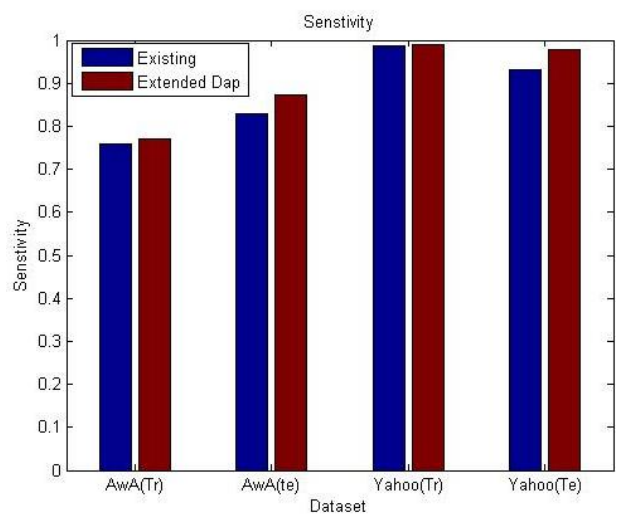

Fig 4: Comparison of Sensitivity

The figure 4 shows the comparison of the sensitivity values for the existing, extended Dap and the proposed algorithm over described datasets. The improvement in the sensitivity can be analyzed by the graph.

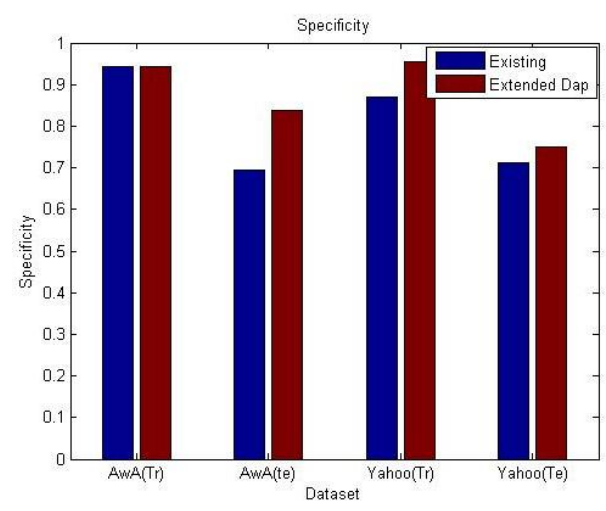

Fig 5: Comparison of Specificity

The figure 5 shows the comparison of the specificity values for the existing, extended Dap and the proposed algorithm over described datasets. The improvement in the specificity can be analyzed by the graph. The improvement in the classification accuracy and other parameters can be easily determined by examine the table and the graph. 


\section{CONCLUSION}

This work extends the Dap by using a gain based tree classification which improves the accuracy as well as the sensitivity and the specificity of the classification. The classification accuracy and the sensitivity, specificity has been analyzed on two datasets AwA and the yahoo pascal dataset. The analysis clarifies that the extended Dap performs better as compare to the existing work. The further extension of the work has been done by using the swarm intelligence with SVM classifier.

\section{REFERENCES}

[1] D. G. Lowe, "Distinctive image features from scaleinvariant key-points", International Journal on Computer Vision (IJCV), vol. 60, no. 2, 2004.

[2] C. H. Lampert, H. Nickisch, and S. Harmeling, "Learning to detect unseen object classes by betweenclass attribute transfer", in IEEE Conference on Computer Vision and Pattern Recognition (CVPR), 2009.

[3] David G. Lowe et. al. ,"Object Recognition from Local Scale-Invariant Features", Proc. of the International Conference on Computer Vision, Corfu (Sept. 1999).

[4] N. Dalal and B. Triggs, "Histograms of oriented gradients for human detection", in IEEE Conference on
Computer Vision and Pattern Recognition (CVPR), 2005.

[5] N. Kumar, A. Berg, P. Belhumeur, and S. Nayar "Describable visual attributes for face verification and image search", IEEE Transactions on Pattern Analysis and Machine Intelligence (PAMI), vol. 33, no. 10, pp. 1962-1977, 2011.

[6] K. Yanai and K. Barnard, "Image region entropy: a measure of visualness of web images associated with one concept", in Proceedings of the 13th annual ACM international conference on Multimedia. ACM, 2005, pp. 419-422.

[7] A. F. Smeaton, P. Over, and W. Kraaij ,"Evaluation campaigns and trecvid", in ACM Multimedia Information Retrieval, 2006.

[8] D. Parikh and K. Grauman, "Interactively building a discriminative vocabulary of nameable attributes," in IEEE Conference on Computer Vision and Pattern Recognition (CVPR), 2011, pp. 1681-1688.

[9] V. Sharmanska, N. Quadrianto, and C. H. Lampert, "Augmented attribute representations", in European Conference on Computer Vision (ECCV), 2012. 